\title{
Research on the Analysis and Prediction Model Building of Mobile User Behaviors
}

\author{
Haiyan Luo ${ }^{1, a}$, Yong Yang ${ }^{1, b}$, Jue Wang ${ }^{1, c}$, Hailong $Y^{2, d}$ \\ ${ }^{1}$ College of Information and Electrical Engineering, Shenyang Agricultural University, Shenyang, \\ 100866, China \\ ${ }^{2}$ China Mobile Communications Corporation Liaoning Co., Ltd, Shenyang, 110179, China \\ a423233233@qq.com, byangsyau@163.com, 'kelly_wang13@126.com, \\ dyuhailong@In.chinamobile.com
}

Keywords: mobile user behaviors; decision tree; personalized; behavior prediction

\begin{abstract}
The personalized user operation is the mainstream of the mobile application development in the future, which is based on the accurate prediction for mobile user behaviors. Currently, the mobile applications cannot provide specific mobile application operating procedures according to the behavioral characteristics of different users, resulting in inferior user experience for the mobile applications. The lack of personalized experience may affect the mobile user experience for mobile applications and services. This paper proposed a method to build a decision tree-based model to predict user behaviors. Results shows, this method could greatly improve the accuracy and reliability of the prediction during the construction of analysis model based on the mobile users' behavior.. It is conducive to achieving the personalized customization of mobile applications. It can promote the power of competition in the marketing of enterprises.
\end{abstract}

\section{Introduction}

Currently, in mobile applications, the development of various function flows is based on the inherent habitual patterns. In the process of development, engineers conduct the design simply by virtue of development experience and local market research [1], so the all mobile users are using the same operating procedures and designers fail to provide specific operational processes based on behavioral characteristics of different users, resulting in inferior user experience for the mobile applications [2]. The lack of personalized experience may affect the mobile user experience for mobile applications and services [3]. To solve this problem, this paper proposed a method to build a decision tree-based model to predict user behaviors from the perspective of mobile users' personalized experience. This method can be used to achieve accurate prediction of mobile user behaviors and provide reliable predictive data support for the personalized customization and experience [4]. It is conducive to further promoting the service quality of mobile applications.

\section{Data Collection of Mobile User Behaviors}

Data collection is the basis to predict mobile user behaviors. The data collection in the predictive model of this paper is based on the data collection framework, as shown in Fig. 1. First, collect data from the mobile terminal of mobile users [5], which mainly includes the perception information and operational information of user device. Then collate the collected information and transmit the data to the user model training database of the mobile user behavior model by the local storage, aiming to provide data support for the follow-up mobile user behavior model. 


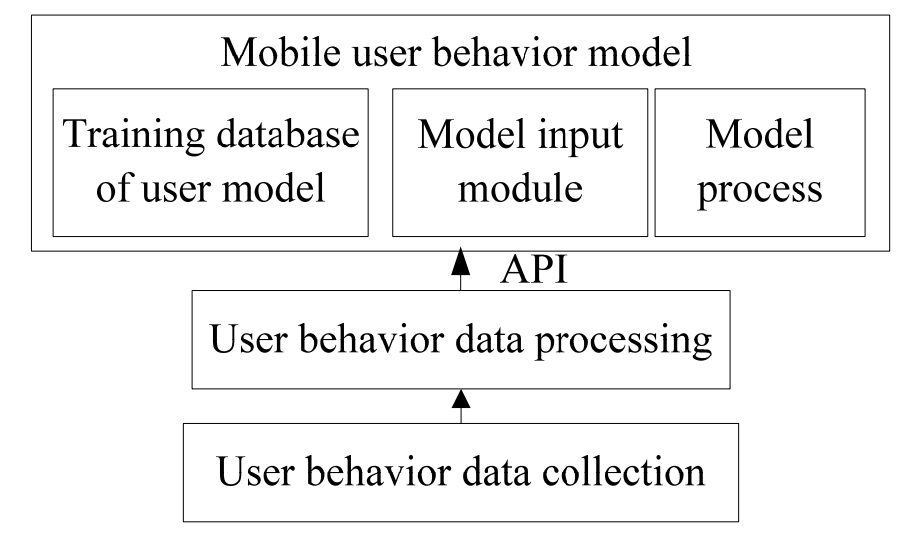

Fig. 1. Data collection model of mobile user behaviors

\section{Decision Tree (DT) Algorithm}

The analysis and prediction model of mobile user behaviors in this paper is based on the decision tree algorithm. It is a typical machine learning classification algorithm, which can be used to derive an appropriate representation method of the decision tree from a class of disordered random sample data. It is commonly used in various predictive models and classifiers [6]. In this model, the decision tree is usually used to predict the mobile user behaviors, as shown in Fig. 2.

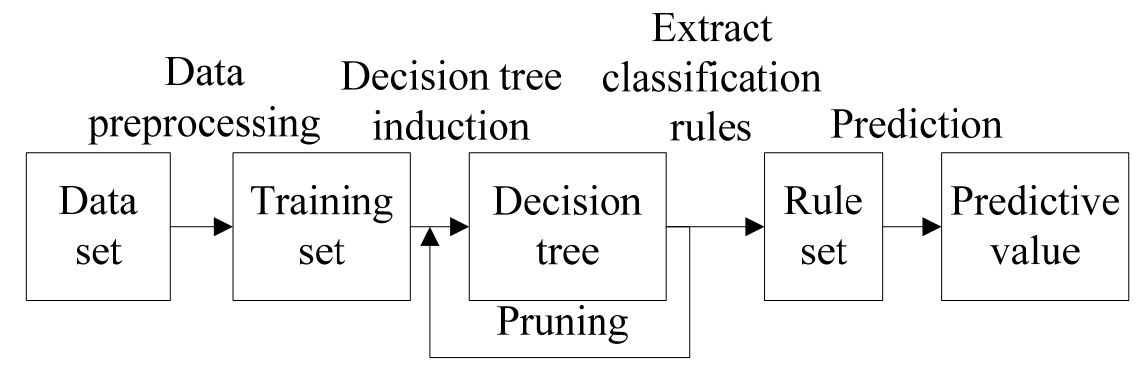

Fig. 2. Flowchart of decision tree algorithm

The execution of the decision tree includes the following two steps:

(1) Draw training samples from the training database of mobile user behaviors to establish decision tree model, namely, machine learning. Its implementation typically includes decision tree building and decision tree pruning [7].

(2) Classify these training samples based on the above decision tree model to determine the direction of each branch [8].

To build the decision tree, the attribute selection metric is used to determine the topology between different characteristic attributes, as shown in Fig. 3. First, build node $\mathrm{N}$ by the sample collection $S$ and then judge it, and finally select the best attribute to split $S$ to get the complete decision tree.

Based on the above principles of decision tree, the decision tree building in the analysis process of mobile user behaviors is as shown in Fig. 4. This decision tree is to predict the operation of a mobile user. Here, three steps are used as an example, access to a web page, launch the game, and online shopping. These three operations represent three categories in the decision tree. Meanwhile, that the location of mobile users is less than or equal to 30 and not indoor is regarded as a set of classification rules, and based on this rule, the "indoor" and "outdoor" are used to represent the characteristics of the decision tree. The implementation process of the decision tree still requires accurate analysis and appropriate classification characteristics, which may affect its prediction efficiency for mobile user behaviors. Thus, the whole decision tree can be used to obtain a set of rules and then predict the mobile user behaviors by the location information. This is only part of the method which also takes into consideration of how the user behaved last time and regards it as a basis for prediction. Its prediction mode is similar to this model, but the feature extraction is conducted based on the operation category, and then predict the next operation. 


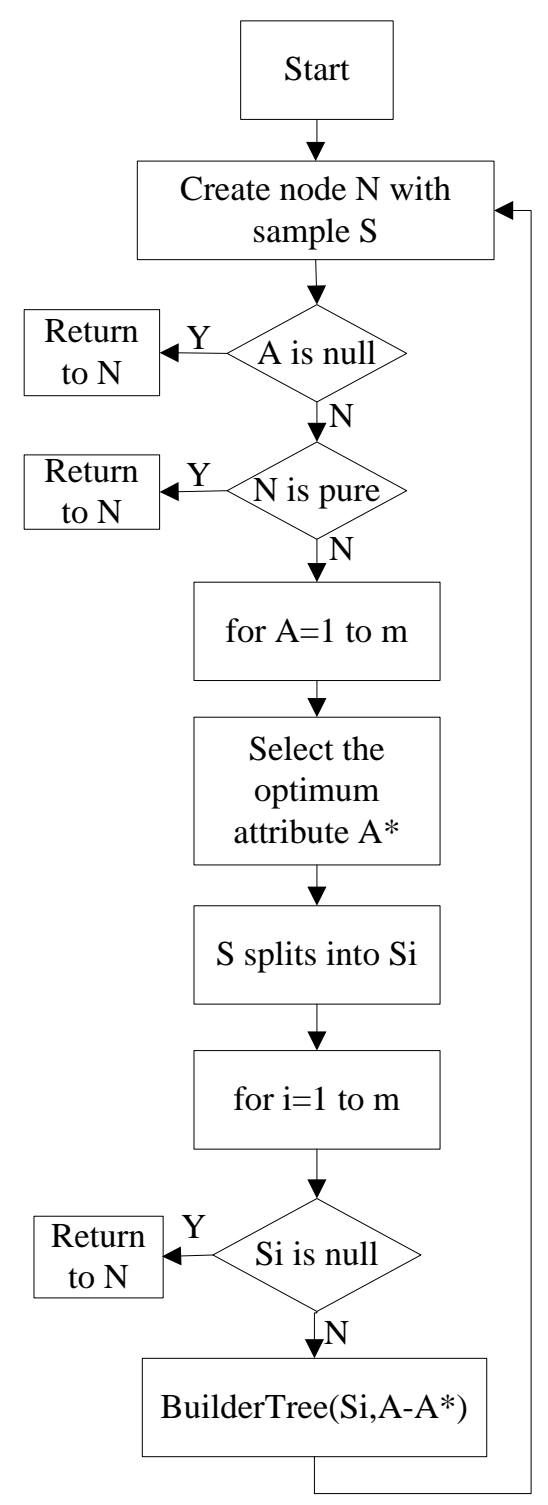

Fig. 3. Flowchart of decision tree building

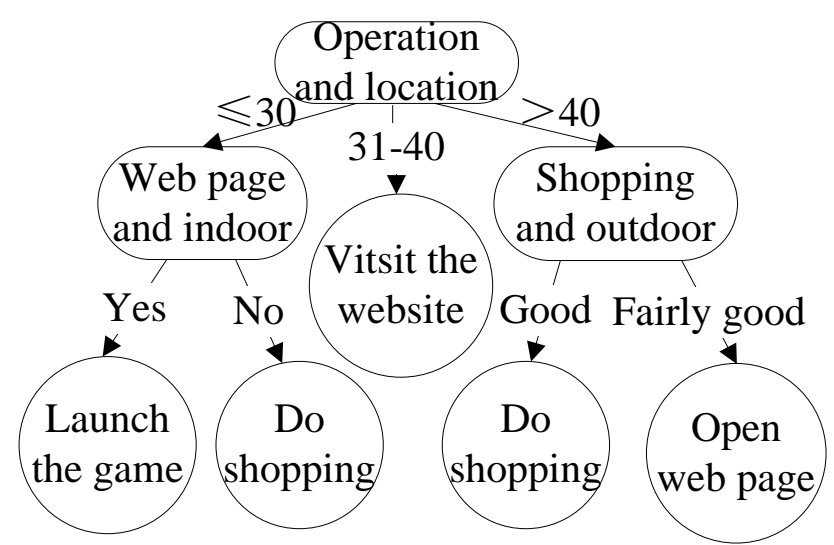

Fig. 4. Mobile user behaviors prediction model based on the decision tree

\section{Overall Design of the Analysis Model for Mobile User Behaviors}

The above chapters have described the data collection of mobile user behaviors in detail and presented specific data collection model. On this basis, the data collected by this model is regarded as the training sample to process and obtain the normal behaviors of mobile users as the input of the model. Meanwhile, the perception information of users is regarded as the condition of the model to 
predict the mobile user behaviors for the next step [9], and then output prediction results. The feedback information, an important basis to improve operational processes of mobile applications, and adaptive adjustment modules of mobile applications form a complete feedback, to achieve the adaptive adjustment of the operational process by the prediction of mobile user behaviors of mobile applications. Therefore, it could provide personalized service for different mobile users.

In this paper, the design process of mobile user behavior model is based on the decision tree, and the behavior graphical model is used to simulate the mobile user behaviors, as shown in Fig. 5. The whole mobile user behaviors graph includes two parts, collection of user behaviors and decision tree set, wherein the former one is mainly used to describe a collection of mobile user behaviors, such as access to Web pages, launch the game and do shopping on line, etc. While the latter one is mainly used to predict the subsequent operation of this behavior.

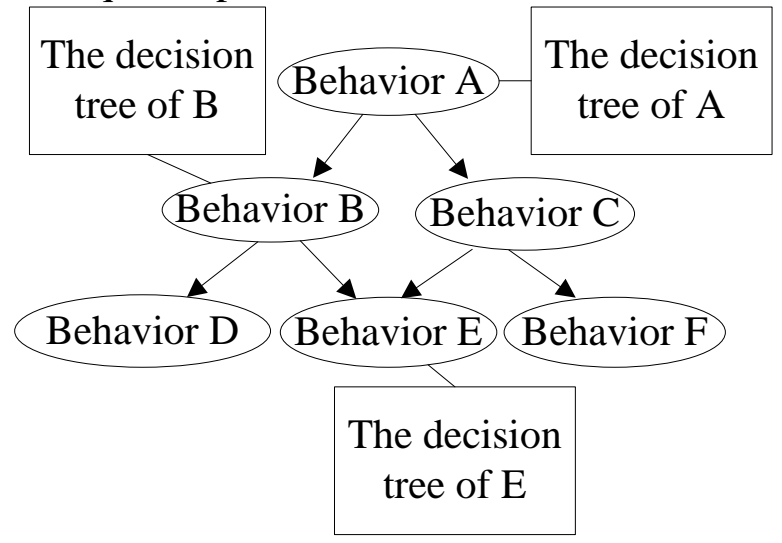

Fig. 5. Structure of mobile user behavior graphical model

\section{Operational Process Design of Mobile User Behavior Model}

The operation procedures of mobile user behaviors model in this paper are as shown in Fig. 6 .

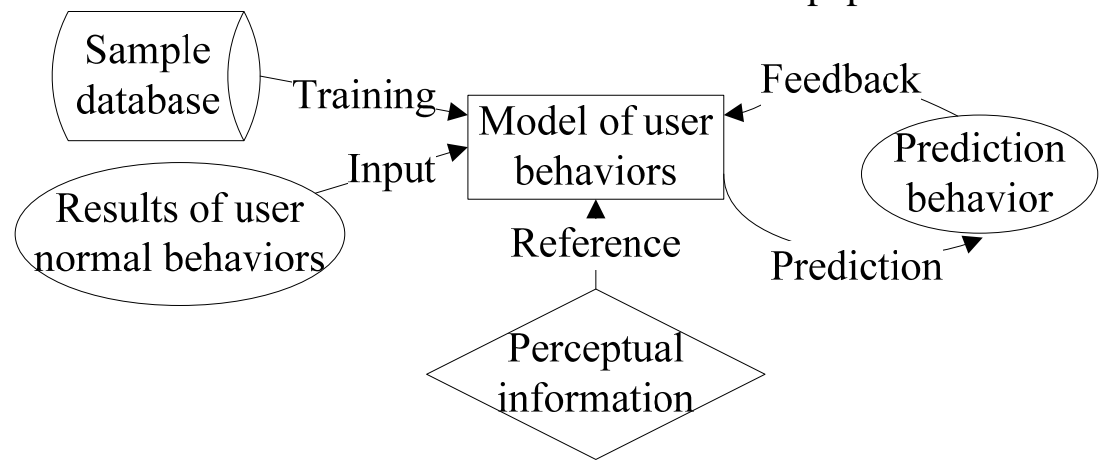

Fig. 6. Operating process of mobile user model

First, set the location information of mobile user by the configuration file [10], and then set mobile user operation corresponding to different predictions. Secondly, when the collected data of mobile users reaches a certain threshold value, that is, it could meet the requirement of decision tree, start the learning mode based on the decision tree, including the initialization of mobile user behaviors, the generation of decision tree and its pruning process, etc. Finally, save the samples for subsequent decision-making. On this basis, if mobile user behaviors change, the system will automatically adjust the user behaviors and then start the decision tree module to conduct the prediction with adaptive adjustment [11]. By the predicted feedback information, the system could control the operation of users. Set different operation processes according to the predictive results, thereby achieving customized and personalized service, so as to effectively improve the quality of user operation services. 


\section{Conclusion}

This paper built the analysis and prediction model for mobile user behaviors based on the decision tree. Then it presented the overall design and implementation process of the model, and analyzed the modeling process, establishment of the decision tree in detail. Finally, it analyzed the expected effect of the model as well as the accuracy and feasibility of the mobile user behavior model from the theoretical viewpoint. This model could accurately and effectively predict the mobile user behaviors and is of great significance to improve the personalized operation and service of mobile applications.

\section{Reference}

[1] H.L. Di, "3G Subscriber Behaviors Model and Empirical Research based on Data Mining for the Precise Marketing," Beijing University of Posts and Telecommunications, 2012.

[2] X.Y. Li, "A Study of Mobile Environment Oriented Efficient Context Data Mining and Energy Efficient Sensing," University of Science and Technology of China, 2012.

[3] Q.L. Zhou, "Research on the Key Technology of the Analysis System of Mobile User Online Behaviors ," Beijing Jiaotong University, 2014.

[4] J.M. Wang, "Analysis of the Mobile Search Behaviors based on User Log," Library and Information Service, vol. 57, no. 19, pp. 102-106, 2013.

[5] M. Xiao, "Research and Implementation of Mobile Community Discovery Method based on the Mobile User Behaviors," Beijing University of Posts and Telecommunications, 2013.

[6] W. Li, "Analysis of Mobile Internet User Behaviors," Beijing University of Posts and Telecommunications, 2013.

[7] P. Wang, "Key Technology of Web Users' Behaviors Analysis Based on Dynamic Behaviors Profile Database," Computer Technology and Development, vol. 19, no. 02, pp. 20-23, 2009.

[8] Y.C. Shi, "Adaptive Learning Approach of Contextual Mobile User Preferences," Journal of Software, vol. 23, no. 10, pp. 2533-2549, 2012.

[9] M. Xiao, "A Circuits Merging Community Discovery Algorithm Based on Mobile User Behaviors," Journal of Electronics \& Information Technology, vol. 34, no. 10, pp. 2369-2374, 2012.

[10] S.F. Gong, "Research on the Discovery Algorithm of Dynamic Behaviors based on Mobile User Behaviors and Its Implementation," Beijing University of Posts and Telecommunications, 2014.

[11] Z. Guo, "Comprehensive Optimization of Wireless Communication Networks based on Mobile User Behaviors," Huazhong University of Science and Technology, 2011. 